\title{
Kernos
}

Revue internationale et pluridisciplinaire de religion grecque antique

3| 1990

Varia

\section{Mantique et télestique dans le Phèdre de Platon}

\section{Omer Ballériaux}

Édition électronique

DOI : $10.4000 /$ kernos.968

ISSN : 2034-7871

\section{Éditeur}

Centre international d'étude de la religion grecque antique

Édition imprimée

Date de publication : 1 janvier 1990

ISSN : 0776-3824

\section{Référence électronique}

Omer Ballériaux, « Mantique et télestique dans le Phèdre de Platon », Kernos [En ligne], 3| 1990, mis en ligne le 19 avril 2011, consulté le 19 avril 2019. URL : http://journals.openedition.org/kernos/968 ; DOI : 10.4000/kernos.968 


\section{MANTIQUE ET TÉLESTIQUE DANS LE PHÈDRE DE PLATON}

Platon, dans le Phèdre, 248d-e, répartit les conditions humaines en neuf classes, qu'il énumère, par ordre de dignité décroissante, en partant de la plus noble, la condition de philosophe, pour aboutir à la plus vile, la condition de tyran. Au cinquième rang, à égale distance entre les deux extrêmes, se situe une existence que Platon appelle $\mu \alpha v \tau \iota$ kòv Bíov

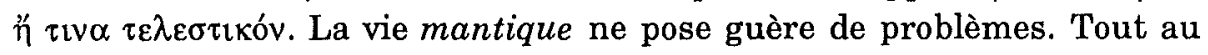
plus s'est-on parfois demandé pourquoi le devin occupe une place aussi modeste dans la hiérarchie platonicienne. Mais à quoi peut bien correspondre, dans la société grecque des Ve et IVe siècles, cette vie

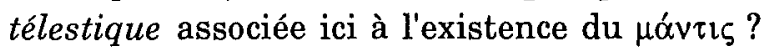

Les traducteurs du Phèdre se montrent, à cet endroit, bien hésitants. Fowler (Loeb Classical Library) traduit the life of a prophet or someone who conducts mystic rites, Hackforth, the life of a mystery-priest. Une existence consacrée à quelque forme d'imitation, lisons-nous sous la plume de Robin (Belles Lettres). Une vie de praticien des initiations, disent Robin-Moreau dans la Bibliothèque de la Pléiade, et la dernière en date des traductions françaises, celle de Luc Brisson, reprend presque textuellement cette formule. Tout cela est assez flou et devient même contradictoire lorsque M. Meunier parle de la vie d'un initiateur tandis que P. Vicaire évoque une vie d'initié. Les dictionnaires, s.v. $\tau \varepsilon \lambda \varepsilon \sigma \tau \iota \kappa o ́ s$, ne nous sont pas d'un grand secours. Le Thesaurus propose initiativus, initiatorius, Liddell-Scott-Jones, connected with magic rites, Bailly, d'initiation aux mystères, propre à initier ${ }^{1}$. C'est que le

1 J'avais un instant envisagé de traiter de la télestique platonicienne en partant de l'étude sémantique du mot $\tau \varepsilon \lambda \varepsilon \sigma \tau \imath$ kós. Mais, entre autres difficultés qui se seraient présentées, il eût fallu aborder l'épineuse question des rapports entre

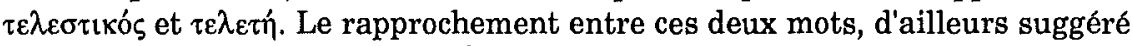

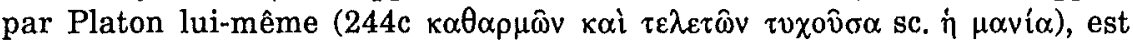
évidemment ce qui a amené lexicographes et traducteurs à parler d'initiation et de mystères. G. CASADIo ayant très judicieusement soulevé le problème dans la brève discussion qui suivit mon intervention, quelques remarques s'imposent.

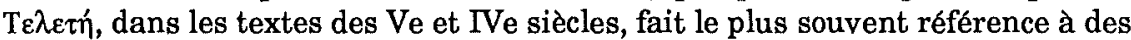

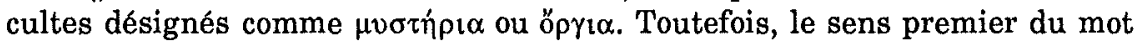
semble avoir été plus général. Les féminins en $-\tau \bar{\alpha}-/-\tau \eta$ - forment un petit groupe de mots qui jouent le rôle de noms d'action (P. CHANTRAINE, La dérivation des mots en grec ancien, Paris, 1933, p. 301) et dès lors, A. BRELICH, Gli eroi greci, Rome, 1958, p. 119 était en droit de voir en $\tau \varepsilon \lambda \varepsilon \tau \eta \dot{~ s e m p l i c e m e n t e ~ i l ~}$ "compimento" di un'azione di culto. Cf. G. CASADIo, Per una indagine storico- 
mot $\tau \varepsilon \lambda \varepsilon \sigma \tau \iota \kappa o ́ \varsigma$ n'est pas attesté avant Platon et que, chez cet auteur, il n'apparaît que deux fois, et les deux fois dans le Phèdre, dans le passage que je viens de citer et, en $265 \mathrm{~b}$, lorsque, prétendant récapituler ce qu'il a dit plus haut des quatre délires qui nous sont accordés par une grâce divine, Socrate en attribue les inspirations à Apollon, à Dionysos, aux Muses, à Aphrodite et à Éros et qualifie ces émínvotoı respectivement de

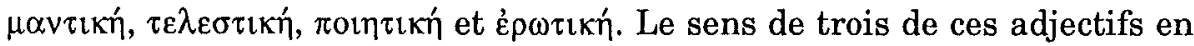
- - кó $\varsigma$ est parfaitement clair parce que nous reconnaissons sans peine les

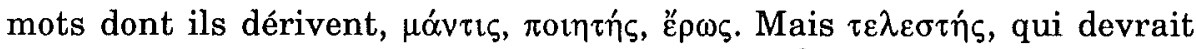
désigner l'homme en proie au délire télestique ${ }^{2}$, ne se trouve pas chez Platon et, sauf erreur, n'est attesté avant lui que dans une inscription, datant de \pm 500 avant J.-C. et trouvée à Olympie. Sous la forme dialectale $\tau \varepsilon \lambda \varepsilon \sigma \tau \dot{\alpha}$, le mot, opposé à $F \varepsilon ́ \tau \alpha \varsigma$, simple citoyen, y signifie magistrat, personne qui exerce une charge publique $(\tau \dot{\lambda} \lambda \circ \varsigma)^{3}$.

Revenons-en au Phèdre. La première idée qui se présente à l'esprit est que, pour comprendre le mot télestique, il suffit de se reporter au passage dont $265 \mathrm{~b}$ se donne pour la récapitulation, soit donc en $244 \mathrm{~d}-\mathrm{e}$, là ou Platon décrit, mais sans la nommer, une folie qui, n'étant ni celle du

religiosa sui culti di Dionisio in relazione alla fenomenologia dei misteri, II, in SMSR, 49 (1983), p. 124-126 et G. SFAMEni Gasparro, Ancora sul termine TE $\Lambda$ ETH, in Filologia et forme letterarie. Studi offerti a F. Della Corte, V, Urbino, 1987, p. 137-152. Oserais-je suggérer une voie d'exploration qui me

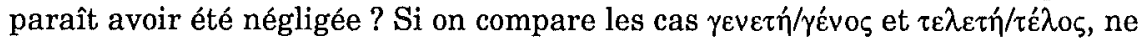
pourrait-on conclure que, de même que үeverí est l'instant précis de la

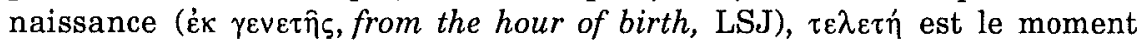

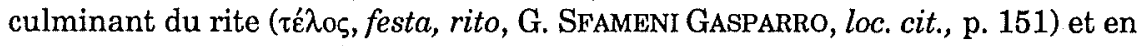
est arrivé, tout naturellement, à désigner, dans un culte à mystères, le rite par excellence, l'initiation?

2 En 265d, à l'exception de L. BRISSON qui parle d'une inspiration initiatique, les traducteurs anglais et français du Phèdre sont unanimes et rendent $\tau \varepsilon \lambda \varepsilon \sigma \tau u$ ó́ par mystic, mystique. L. ROBIN et J. MOREAU précisent dans une note : en prenant cet adjectif au sens littéral : l'inspiration qui préside aux mystères et aux initiations (Platon, CEuvres complètes, II, Paris, 1964 [Bibl. de la Pléiade], p. $1420, n$. 3). Tout n'en est pas plus clair. Il va de soi que l'on ne peut, pour déterminer l'acception du mot chez Platon, recourir aux nombreux emplois de $\tau \varepsilon \lambda \varepsilon \sigma \tau \imath$ xós et de $\tau \varepsilon \lambda \varepsilon \sigma \tau \dot{n} \zeta$ dans les textes de l'antiquité tardive et, plus particulièrement, dans les œuvres des néoplatoniciens. Cf. mon article

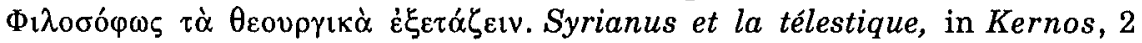
(1989), p. 13-25.

3 F. Solmsen et E. FraenKel, Inscr. graecae ad illustrandas dialectos,

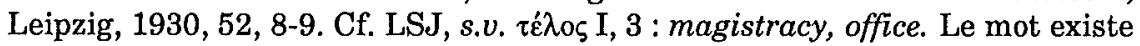
déjà dans le mycénien : tereta est le nom d'un groupe social dont le sens précis est discuté (P. Chantraine, Dict. étymol. de la langue grecque, IV, 1, Paris, 1977, p. 1102). 
devin, ni celle du poète, ni celle de l'amant, ne peut être que le délire de

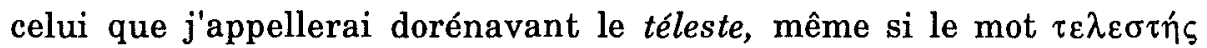
n'est jamais utilisé par Platon. Ce délire est décrit en une longue phrase de huit lignes, qui est, hélas, d'une rare difficulté. Wilamowitz assurait qu'il n'en avait trouvé nulle part une explication qui le satisfit et qu'il ne pouvait lui-même en proposer une qui mît un terme à sa perplexitét Pour invoquer un témoignage plus récent, L. Brisson, il y a une quinzaine d'années, parlait d'un texte très controversé et $d u$ clair obscur qui dérobe les contours de pratiques religieuses plus ou moins marginales ${ }^{5}$. De Lobeck au Père des Places, ceux qui se sont efforcés de préciser qui sont les religious experts ${ }^{6}$ dont il est ici question nous dépeignent les télestes sous des aspects bien divers. Il faudrait citer ce qu'en ont dit E. Rohde, A.E. Taylor, Fr. Pfister, mon maître A. Delatte, son élève J. Croissant, Linforth, dont l'article, publié en 1946, a fait longtemps autorité en la matière, le Père Festugière, De Vries, et j'en passe ${ }^{7}$. Les interprétations de ces érudits, tous éminemment respectables, ont, en dépit de leur divergences, un trait commun qui me paraît devoir éveiller la méfiance. Platon divise ici l'ensemble formé par ceux qui ont obtenu la grâce d'être fou de par les dieux en quatre sous-ensembles, dont trois sont très normalement constitués de nombreux éléments - devins, poètes, amants - que nous pouvons, à notre gré, prendre parmi les contemporains de Socrate ou de Platon, ou bien encore choisir dans l'histoire et la littérature grecques. Les interprétations dont j'ai cité les auteurs nous demandent toutes de considérer le quatrième sous-ensemble, celui des télestes, comme le seul

4 WILAMOWTT, Platon, Berlin, $1959^{5}$, p. 322, n. 3; p. 375, n. 1.

5 L. BRISSON, Du bon usage du dérèglement, in J.P. VeRNANT et al., Divination et rationalité, Paris, 1974, p. 224-225.

6 L'expression est de R. HACKFORTH, Plato's Phaedrus, Cambridge, 1952 (nombr. réimpr.), p. 84.

7 LoBECK, Aglaophamus, Königsberg, 1829 (repr. Darmstadt, 1961), p. 636-637; STAllbaum, Platonis Opera, IV, 1, Gotha, 18572, p. 82-83; E. Rohde, Psyché, éd. franç. par A. REYMoND, Paris, 1928, p. 304, n. 5; WILAMOWITz, op. cit.; Fr. PFISTER, Der Wahnsinn des Weihepriesters in Cimbria, Dortmund, 1926, p. 5562; A.E. TAYLOR, Plato, the man and his work, Londres, 19293, p. 305-306; J. Croissant, Aristote et les mystères, Liège, 1932, p. 18; A. DelatTe, Les conceptions de l'enthousiasme chez les philosophes présocratiques, Paris, 1934, p. 69 et 71; I.M. LINForTH, Telestic madness in Plato, Phaedrus 244 D-E, Berkeley, 1946 (Univ. of California Publ. in Class. Philology, 13, 6), p. 162-173; A.J. FesTUGièe, c. r. du précédent in REG, 59-60 (1946-1947), p. 494-495; É. DES Places, art. Extase, in Dictionnaire de spiritualité, IV, Paris, 1961, col. 20612062; DE VRIES, A commentary on the Phaedrus of Plato, Amsterdam, 1969. 
à ne comprendre que quelques rares éléments, que nous devrions aller chercher dans un lointain passé, Mélampe, Épiménide, Pythagore pour les uns, pour les autres quelques Labdacides, Atrides ou Alcméonides, pour d'autres enfin des fondateurs de religions ou des créateurs de cultes orgiastiques, dont on devine bien qu'ils ne sont pas légion.

Semblable reproche ne peut en tout cas s'adresser à W. Burkert qui nous présente les télestes comme les prêtres ambulants d'une religion caractérisée par des rites de purification dont il découvre le modèle dans les incantations assyriennes. Pour Burkert, l'introduction de cette cathartique, au VIIe siècle, est la première des vagues d'orientalisme qu'a connues l'histoire de la religion grecque ${ }^{8}$. Permettez-moi de laisser de côté ce qu'a éventuellement d'oriental la télestique, non point en raison de cette réaction naturelle du philologue classique qui, comme le suggère Burkert ${ }^{9}$, voulant toujours griechische Gebräuche durch griechische Worte erklären ${ }^{10}$, se montre réticent dès qu'on lui propose d'expliquer la Grèce par des influences extérieures. Beaucoup plus simplement, ignorant tout de Sumer et d'Akkad, je n'entends pas, tel le cordonnier, aller ultra crepidam. Je tenterai donc de cerner l'image du téleste en ayant recours aux seules ressources de la littérature grecque.

La phrase qui désespérait Wilamowitz se compose de deux parties, une brève protase qui décrit un certain mal, dont l'apodose nous explique que la télestique a trouvé le moyen d'en débarrasser ceux qui en sont

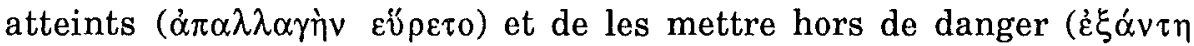
$\left.\dot{\varepsilon} \pi \circ i_{\eta} \sigma \varepsilon v\right)$. Comme L. Brisson l'a bien observé, la thérapeutique, les rites cathartiques sont enveloppés d'obscurité. Nous nous tournerons donc vers l'exposé pathologique de la protase.

Pour désigner le mal contre lequel la télestique a trouvé une parade,

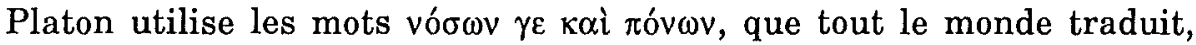
naïvement, dirais-je, par «les maladies et les épreuves» ${ }^{11}$. Mais c'est

8 W. BURKERT, Itinerant Diviners and Magicians : A neglected element in cultural contacts, in The Greek renaissance of the 8th century B.C., Proceedings of the 2d Symposium of the Swedish Institute in Athens, ed. by R. HÄGG, Göteborg, 1983 (Acta Instituti Atheniensis Regni Sueciae, Ser. in $4^{\circ}$, 30), p. 115119. Cf. ID., Greek Religion, Cambridge (Mass.), 1985, p. 296-297 (=trad. angl. par J. RAFFAN de Griechische Religion der archaischen und klassischen Epoche, Stuttgart, 1977).

9 W. BURKERT, ГOH, Zum griechischen Chamanismus, in RhM, 105 (1962), p. 37-38.

10 L'expression est de O. KeRN, Religion der Griechen, I, Berlin, 1926, p. 13.

11 C'est dans le sens tout ordinaire de maladies et de travaux que sont employés

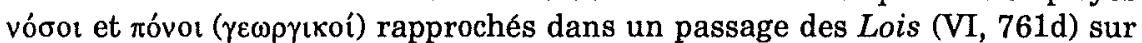




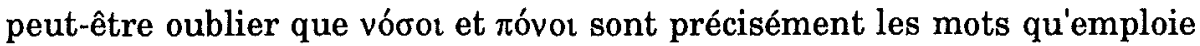
Oreste, dans la tragédie d'Euripide qui porte son nom, pour parler des maux qu'il endure pour avoir versé le sang maternel et qu'il refuse de

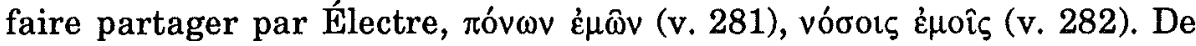

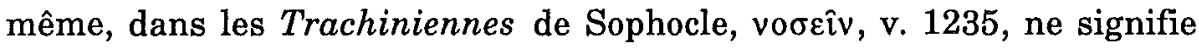
pas être malade au sens habituel du terme. Héraclès mourant demande à son fils d'épouser Iole, et Hyllos de répondre qu'à sa place, nul ne le ferait à moins d'être égaré, rendu fou par les mauvais génies.

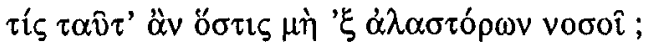

Les vófol du Phèdre, pas plus que les accès d'Oreste, ne sont donc point, comme le voulait $\mathrm{A}$. Delatte, des névropathies héréditaires. $\mathrm{Ce}$ sont peut-être, comme le dit Burkert, des troubles psychosomatiques ${ }^{12}$, mais en tout cas, pour Sophocle, pour Euripide, pour Platon et pour leurs contemporains, ces égarements, ces accès de folie sont dus à une malédiction divine personnifiée par les Érinyes ou les Alastores. Nóoor et róvor sont, en fait, ce qui, dans l'arsenal des châtiments dont disposent les dieux, correspond, lorsqu'il s'agit de punir des individus ou des

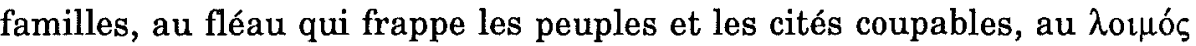
dont Marie Delcourt nous a montré qu'il n'y faut point voir, si j'ose dire, une peste quelconque ${ }^{13}$.

Socrate précise lui-même que ces égarements et ces épreuves sortent

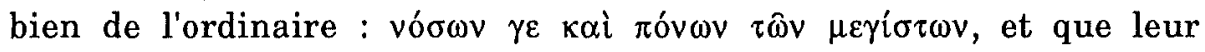
origine mystérieuse est à rechercher dans des courroux divins d'ancienne date, ne s'observant d'ailleurs que dans certaines lignées,

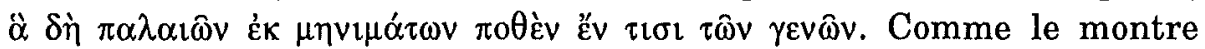
la place inhabituelle de la préposition $\dot{\varepsilon} \kappa, \pi \alpha \lambda \alpha \iota \hat{\omega} v \dot{\varepsilon} \kappa \mu \eta \nu \iota \mu \dot{\alpha} \tau \omega v$ est

lequel Mme S. GEORGoudi a attiré mon attention lors du Colloque de Liège. J'ai sans doute eu tort de faire porter mes recherches uniquement sur vóoor et l'on pourrait peut-être aussi trouver des emplois de $\pi$ óvol où ce terme revêt une acception toute particulière.

12 W. BURKeRT, Itinerant Diviners..., p. 116 : in other words, they offer cures for psychomatic illness. Cf. ID., Craft versus Sect : the problem of Orphics and Pythagoreans, in Jewish and Christian Self-Definition, III, Londres, 1982, p. 5 : "present ills", "terrible sufferings" such as occur in "certain families", especially «disease»; in modern terms, these are situations of individual crisis, somatic, psychosomatic, psychic and social disturbances.

13 M. DELCOURT, Stérilités mystérieuses et naissances maléfiques dans l'antiquité classique, Liège-Paris, 1938, p. 14 et sq. 
évidemment une citation "poétique» et, depuis Buttmann au moins ${ }^{14}$, les commentateurs nous renvoient aux Phéniciennes d'Euripide,

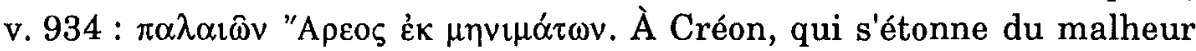
qui les frappe, lui et son fils, Tirésias explique qu'ils expient la faute commise par Cadmos, coupable d'avoir tué le dragon né de la terre. L'Edipe à Colone éclaire parfaitement le sens de $\pi \alpha \lambda \alpha$ ì̀ $\mu \eta v i ́ \mu \alpha \tau \alpha^{15}$,

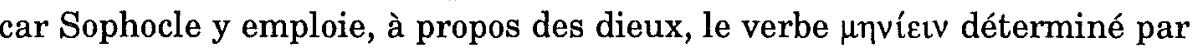

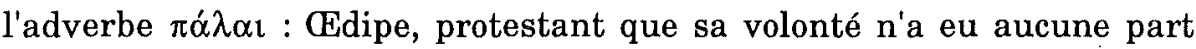
dans les fautes qu'il a commises ă $\kappa \omega v$, en rejette la responsabilité sur les dieux, sans doute irrités d'ancienne date contre sa lignée :

$$
\begin{aligned}
& \theta \text { coîs }
\end{aligned}
$$

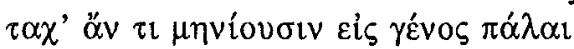

Il y a enfin, chez Eschyle, un troisième passage parallèle. Dans l'Agamemnon, Clytemnestre déclare, pour sa défense, que la mort d'Agamemnon n'est point son œuvre, mais celle de l'antique mauvais

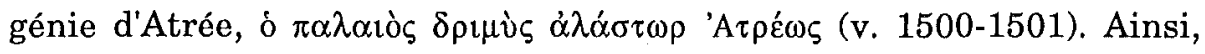
chez les trois grands tragiques, $\pi \alpha \lambda \alpha$ ló $\varsigma$ et $\pi \alpha \dot{\lambda} \lambda \alpha \mathrm{l}$ ne se réfêrent point, dans les trois cas cités, à une quelconque ancienneté, mais précisément au décalage chronologique qui sépare le crime resté impuni d'un ancêtre coupable et le châtiment divin qui atteint son descendant innocent ou qui se dit tel. Le scholiaste d'Aelius Aristide l'a bien vu qui,

14 Cité par StTallbaum, op. cit., p. 82. Ph. Buttmann est l'auteur d'une édition du Phèdre, in usum scholarum, Leipzig, 1839, que je n'ai pu consulter.

15 LSJ propose, comme traduction du pluriel $\mu \eta v^{\prime} \mu \alpha \tau \alpha$, guilt, esp. blood-guiltiness et explique, en alléguant ce passage du Phèdre, $\pi \alpha \lambda \alpha i \alpha \grave{\alpha} \mu$. guilt that cleaves to a family from the sins of their forefathers. Il s'agit bien ici, comme nous allons le voir, de culpabilité héréditaire, mais LSJ confond l'effet et la cause; comme le montrent bien le génitif "Apeos des Phéniciennes et les $\theta \varepsilon o \hat{\varsigma} \pi \alpha \dot{\alpha} \lambda \alpha \iota \mu \eta$ víovotv de l'Edipe à Colone. C'est donc par ressentiments, courroux qu'il faut traduire $\mu \eta v i ́ \mu \tau \alpha$, ou si l'on veut, vindicte, poursuites exercées par les dieux en raison de leur colère. Dans Homère, on ne trouve $\mu \eta \eta v ı \mu \alpha$ qu'au singulier et deux fois seulement. Encore le mot est-il utilisé dans la même formule - $\mu$ í $\tau$ ó $\tau \imath \theta \varepsilon \hat{\omega} v$

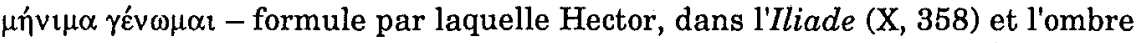
d'Elpénor dans l'Odyssée $(\lambda, 73)$ menacent leur interlocuteur de devenir pour lui - au datif de "désavantage» - ce qui déclenchera la colère divine. Une expression très semblable se rencontre dans une inscription du IIIe ou du IIe

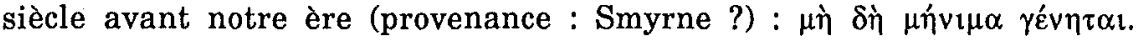
Bromios menace de son courroux ceux qui, sans observer une rigoureuse quarantaine, pénétreraient dans l'enceinte sacrée après avoir exposé un enfant, et prescrit le même délai d'attente pour la femme qui n'a point mené à terme sa grossesse (G. PETzL, Die Inschriften von Smyrna, Teil II, 1, Bonn, 1987, 728, 4). Cf. aussi ANTIPHON, IV, 2, 8 et PAUS., IX, 5, 8. 
son auteur citant textuellement notre phrase du Phèdre dans son

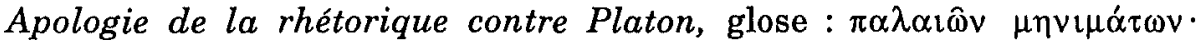

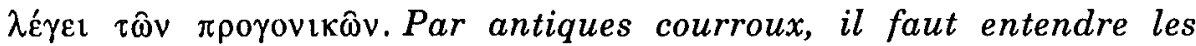
courroux provoqués par les ancêtres ${ }^{16}$ et il donne, à titre d'exemples, Agamemnon payant pour Tantale et Crésus pour Gygès. K. Latte ${ }^{17}$, Dodds 18 et bien d'autres ont mis en évidence cette croyance selon laquelle la culpabilité se transmet héréditairement, croyance qui s'est ancrée dans les conceptions religieuses des Grecs à partir du moment où ils sont passés de la civilisation homérique de la honte, shame-culture, à la civilisation archaïque de la culpabilité, guilt-culture. Que cette croyance était toujours bien vivace à l'époque de Platon, deux exemples suffisent à l'établir. Dans le Busiris, 25, Isocrate s'élève, comme l'avaient fait avant lui Solon ${ }^{19}$ et Théognis ${ }^{20}$, contre cette conviction propre aux Grecs qui prétend reporter sur les enfants innocents la punition des pères coupables et il félicite les Égyptiens de ne point la partager. Dans le Théétète, 173d, pour montrer combien les meilleurs

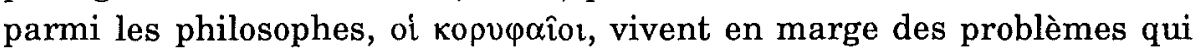
agitent la vie quotidienne des cités, Socrate mentionne, entre autres choses que tout le monde connaît et qu'ignore superbement l'ami de la sagesse, la tare héréditaire que tel ou tel tient de ses ancêtres, paternels

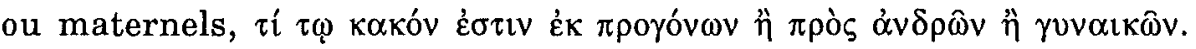
Lorsque donc nous lisons dans le Phèdre que la vindicte divine

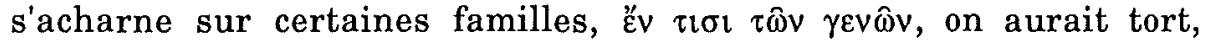
comme le veulent d'aucuns, de restreindre l'application de ce que dit Platon à quelques dynasties légendaires ou à quelques grandes familles de l'histoire ${ }^{21}$, même si ce sont celles-là que, tout naturellement, cite le scholiaste d'Aristide et que met en scène la tragédie.

Nous voyons à présent dans quel cas il convient d'avoir recours aux télestes, mais il nous reste encore à tenter de préciser qui sont ces

16 ARISTIDE e recens. G. Dindorfil, Leipzig, 1829, III, p. 323 (= Sch. au De rhetorica, 14, 13).

17 K. LATTE, Schuld und Sünde in den griechischen Religion, in ARW, 20 (19201921), p. 267 = Kleine Schriften, Munich, 1968, p. 12.

18 E.R. DoDDs, The Greeks and the irrational, Berkeley, 1951, p. 28-63 : Ch. II : «From Shame-Culture to Guilt-Culture» (trad. franç. de M. GIBSoN, Paris, 1965 et 1977).

19 SoLon, fr. 1, 31-32 Diehl.

20 THÉOGNIS, I, 731-752, not. 735-736.

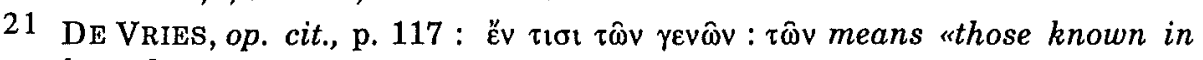
legends". 
personnages qui peuvent sauver les malheureux poursuivis par un courroux divin. Comme W. Burkert l'a bien vu, la clef du problème se trouve dans la République (II, 364b) où Adimante s'en prend à des devins

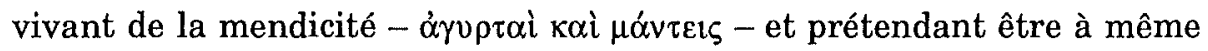
d'apaiser la colère des dieux irrités par la faute qu'a commise celui qui

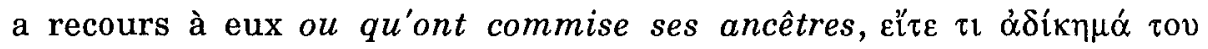

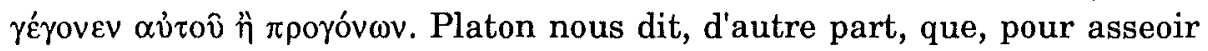
leurs prétentions, ces charlatans produisent un tas de livres dus à Musée et à Orphée (364e), et dès lors, les éditions de la République 22 nous renvoient aux Caractères de Théophraste, XVI, 11, où le superstitieux court, chaque mois, consulter, avec femme et enfants, les orphéotélestes. Il me paraît évident que ceux que le Phèdre nous montre possédés par

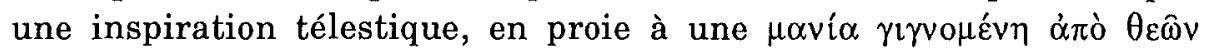
(245b) et délivrant les membres de certaines familles de la malédiction divine qui pèse sur eux en raison du crime impuni d'un ancêtre ne sont autres que les orphéotélestes de Théophraste que l'on consulte en famille, eux-mêmes identiques aux devins de la République qui,

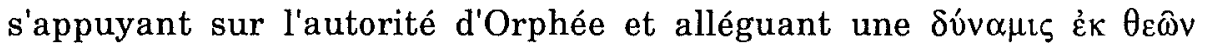
$\pi \circ \rho \iota \zeta o \mu \varepsilon ́ v \eta(364 b)$ interviennent en faveur de gens sur qui pèse une culpabilité personnelle ou familiale. Mais on comprend aisément pourquoi un certain blocage a longtemps - et peut-être inconsciemment - détourné les exégètes d'opérer ce rapprochement entre le Phèdre et la République. Pouvait-on établir un lien entre les frères mendiants de la République et les télestes inspirés du Phèdre, alors que Socrate, dans ce dernier dialogue, nous dit que les folies divines sont accordées pour le

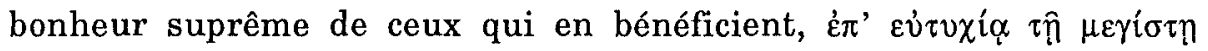
(245b) ? Guthrie, lorsqu'il étudie Orphée et la religion grecque, ne peut cacher son dépit de rencontrer les charlatans de la République et il parle alors de ces faux prophètes comme toute religion en possède 23 . Moulinier, notant le mépris que manifeste Platon à l'égard de certains maîtres ès purifications, se demande : $Y$ aurait-il deux orphismes ${ }^{24}$ ? Pourtant, lorsqu'il s'agit des poètes, ces autres fous de par les dieux, Hackforth est bien contraint de constater que, dans le Phèdre seulement,

22 The Republic of Plato ed. by J. ADAM, Cambridge, 1902, (2d ed. by D.A. REES, Cambridge, 1953 et nombr. réimpr.), p. 80-81; PLATON, La République, Livres IIII, texte établi et trad. par É. CHAMBRY, Paris, 1932, p. 58, n. 1.

23 W.K.C. GUTHRIE, Orphée et la religion grecque, trad. franç. de S.M. GuILlemin, Paris, 1956, p. 28, cf. p. 228; The Greeks and their Gods, Londres, 1968, p. 321.

24 L. Moulinier, Le pur et l'impur dans la pensée des Grecs d'Homère à Aristote, Paris, 1952, p. 138-139. 
leur folie inspirée reçoit une approbation sans réserve - unqualified commendation - tandis qu'ailleurs Platon insiste sur l'aspect négatif de leur «enthousiasme», tenu pour synonyme de non-science ${ }^{25}$ ! Dans

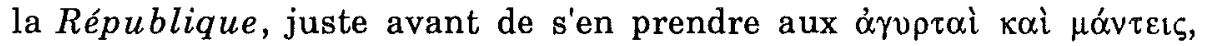
Adimante dénonce avec vigueur et passion les discours des poètes et $P$. Vicaire observe que Socrate, prenant cette attitude à son compte, entreprend tout aussitôt le procès du pouvoir corrupteur de la poésie ${ }^{26}$. Nul n'a pourtant distingué deux espèces de poètes, poètes inspirés du Phèdre et poètes maudits de la République. De même, je tiens pour assuré qu'il n'y a point de différence entre ceux que le Phèdre nous montre favorisés par les dieux d'un délire télestique et ceux qu'Adimante, dans

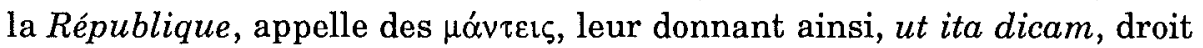
de cité en ce colloque. Dans les siècles de foi, le rôle de ces télestes, de ces devins-exorcistes, était de délivrer de la malédiction divine les membres de certaines familles que l'on disait frappées en raison d'une culpabilité héritée d'un ancêtre. Avec les devins au sens habituel du terme, ces télestes devaient constituer ce que j'appellerais volontiers un clergé, si l'on veut bien bannir de ce mot toute idée de corps constitué, d'ordre, plus encore de caste, mais y voir seulement une manière commode de désigner l'ensemble des gens à qui s'adressent États et particuliers pour régler leurs relations avec la divinité.

Stoqueu, 40

Omer BALLÉRIAUX

B - 4070 AYWAILLE

25 HACKFORTH, op. cit., p. 61.

26 P. VICAIRE, Platon critique littéraire, Paris, 1960, p. 42. 